\title{
Philosophiques
}

\section{Le savoir absolu hégélien, ou comment rentrer chez soi}

\section{Éric Guay}

Volume 26, numéro 1, printemps 1999

URI : https://id.erudit.org/iderudit/004931ar

DOI : https://doi.org/10.7202/004931ar

Aller au sommaire du numéro

Éditeur(s)

Société de philosophie du Québec

ISSN

0316-2923 (imprimé)

1492-1391 (numérique)

Découvrir la revue

Citer cet article

Guay, É. (1999). Le savoir absolu hégélien, ou comment rentrer chez soi. Philosophiques, 26(1), 71-82. https://doi.org/10.7202/004931ar

\section{Résumé de l'article}

Inspiré par la fascinante métaphore hégélienne de la " maisonnette » tirée d’un écrit de jeunesse, ce texte veut montrer sa concrétisation postérieure par le "Savoir absolu » de la Phénoménologie de l'Esprit (1807). On a souvent dit que l'ouvrage de 1807 réconciliait la modernité avec l'ère hellénique. De plus, l'on sait que Hegel considère cette dernière comme le premier moment du savoir, illustrant ce chez-soi auquel les Modernes ont dû renoncer pour acquérir leur propre vision du monde. L'argumentation présentée ici vise à montrer qu'à la manière d'un sujet qui, après un laborieux périple, revient enfin chez soi, c'est bien à un retour en " pays de connaissance " que nous convie cette œuvre célèbre du philosophe allemand. Miracle rendu possible par la découverte d'une circularité qui nous traverse tous ! 


\title{
Le savoir absolu hégélien, ou comment rentrer chez soi
}

\author{
ÉRIC GUAY \\ Département de philosophie \\ Université du Québec à Trois-Rivières
}

\begin{abstract}
RÉSUMÉ. - Inspiré par la fascinante métaphore hégélienne de la «maisonnette » tirée d'un écrit de jeunesse, ce texte veut montrer sa concrétisation postérieure par le «Savoir absolu » de la Phénoménologie de l'Esprit (1807). On a souvent dit que l'ouvrage de 1807 réconciliait la modernité avec l'ère hellénique. De plus, l'on sait que Hegel considère cette dernière comme le premier moment du savoir, illustrant ce chez-soi auquel les Modernes ont dû renoncer pour acquérir leur propre vision du monde. L'argumentation présentée ici vise à montrer qu'à la manière d'un sujet qui, après un laborieux périple, revient enfin chez soi, c'est bien à un retour en « pays de connaissance » que nous convie cette œuvre célèbre du philosophe allemand. Miracle rendu possible par la découverte d'une circularité qui nous traverse tous!
\end{abstract}

\begin{abstract}
Inspired by the fascinating Hegelian's metaphor of the « small house » taken from a youth's work, this paper tends to show its posterior achievement by the « absolute Knowledge » of the Phenomenology of Spirit (1807). It has often been told that the 1807's work settled modernity with Hellenic era. Moreover it is known that Hegel considers the Hellenic era as the first step of the knowledge, demonstrating the home that the Moderns had to renounce to acquire their own point of view of the world. The illustration presented here shows that the Hegel's Phenomenology calls us to a return on «home ground ». The same way as a subject who comes back home after a laborious journey. Miracle enabled by the discovery of a circle going through every one of us !
\end{abstract}

La philosophie est précisément cela : être vraiment chez soi (zu Hause), - que l'homme soit dans son esprit chez lui en terre natale ${ }^{2}$.

Alors qu'il est étudiant au Tübinger Stift, entre 1788 et 1793, le jeune Hegel s'adonne à la rédaction d'un texte interrogeant la possibilité d'accéder, avec l'aide de la religion, à un lieu où l'homme peut arriver à se sentir pleinement chez lui dans le monde. On peut en effet y lire : "la maisonnette (das Häuschen) que l'homme peut alors déclarer sienne, il faut que la religion aide à la bâtir. ${ }^{3}$ » L'image de cette demeure, ce "chez-soi » qui fait aussi figure de " patrie » (einheimisch), traverse l'ensemble des écrits hégéliens, comme en

I. Ce texte, financé par le Fonds FCAR, est une version remaniée d'une communication libre présentée au 65 ${ }^{\mathrm{e}}$ congrès de l'ACFAS tenu à Trois-Rivières du 12 au 16 mai 1997.

2. G.W.F. Hegel, Vorlesungen über die Geschichte der Philosophie I, Sämtliche Werke \# 17, Stuttgart, F. Frommann, 1965, p. 190 ; G.W.F. Hegel, Leçons sur l'histoire de la philosophie I, trad. Garniron, Paris, Vrin, 1971, p. 23.

3. G.W.F. Hegel, Tübinger Fragment, in Theologische Jugendschriften <désormais 
témoignent d'ailleurs les paroles d'un Hegel plus mature présentées en exergue. Mais ces dernières dénotent par contre l'organisation d'un système de pensée qui fait malheureusement défaut aux premières tentatives d'accéder à ce «lieu " où l'on soupçonne que pourrait sereinement "demeurer " l'humanité. On devra attendre près de quatorze ans après la démarche du Fragment de Tübingen, que l'on attribue plus précisément aux années 17921793, pour que le chemin qui conduit à la construction de cette " maisonnette ", transposée en Savoir absolu (das absolute Wissen), nous soit adéquatement montré selon le même impératif religieux, par la publication de la Phénoménologie de l'Esprit ${ }^{4}$. Si le Tübinger Fragment manque incontestablement sa cible, en ne nous permettant pas d'associer le monde moderne à cette « petite maison » où l'on se sent si bien «chez-soi ", c'est en grande partie parce qu'il n'envisage pas encore la modernité comme un " progrès » de la pensée, mais bien plutôt comme un "déclin ". C’est là un écueil qui guette tout homme qui se questionne sur son savoir à partir de la pensée moderne, en jetant un coup d'œil rétrospectif sur la sagesse d'autrefois qui brille en raison de sa fabuleuse simplicité. Attardons-nous-y, en relevant la principale borne qui limite les premières articulations de la pensée hégélienne.

\section{La méconnaissance de la véritable dialectique de la totalité}

Les écrits hégéliens de jeunesse souffrent en plusieurs endroits d'un manque de médiation, d'immédiateté. Mais, nous enseigne Hegel, loin de se révéler comme quelque chose qui serait à proscrire arbitrairement, l'immédiat doit d'abord être assumé avant de s'enrichir pour devenir un moment supérieur de la connaissance ${ }^{5}$. C'est donc un passage obligé de toute pensée de ne pas avoir d'autre choix que de commencer avec ce qui se trouve immédiatement là, et le jeune Hegel n'y a visiblement pas fait exception. Malgré le fait que le Tübinger Fragment expose clairement la nécessité de la médiation de l'immédiateté, ou de " l'expérience jointe à la réflexion ${ }^{6}$ » (Erfahrung verbunden mit Nachdenken) qui nous hisse "au-dessus de la dépendance à l'égard des opinions et des impressions de la sensibilité7 ", ce dernier n'arrive pas à élever la totalité de son

cité : Tüb.>, Frankfurt, 1966, p. 17 ; R. Legros, Le fragment de Tübingen, in Le jeune Hegel et la naissance de la pensée romantique <désormais cité : Frag.>, Ousia, 1980, p. 280. Pour une étude exhaustive du Tübinger Fragment et de la pensée du jeune Hegel, le lecteur lira avec attention l'ouvrage de R. Legros mentionné ci-haut, ainsi que J. Taminiaux, La nostalgie de la Grèce à l'aube de l'idéalisme allemand, Martinus NijHoff, La Haye, 1967.

4. La Phénoménologie de l'Esprit (Phänomenologie des Geistes) fut livrée au public le 29 mars 1807. G.W.F. Hegel, Phänomenologie des Geistes <désormais cité : Ph.G.>, Gesammelte Werke \# 9 , Hamburg, Felix Meiner, 1980 ; G.W.F. Hegel, Phénoménologie de l'Esprit <désormais cité : Ph.E.>, trad. Labarrière/Jarczyk, Paris, Gallimard, 1993.

5. Le concept hégélien d' «Aufhebung», qui exprime l'unité-tripartite des verbes nierconserver-dépasser, illustre bien ce mouvement de transformation de l'immédiateté.

6. Tüb., p. 15 ; Frag., p. 277.

7. Idem. 
discours à ce niveau conceptuel. L'on ne doit pas s'étonner de ce constat, de la part d'un auteur qui, à cette époque, affirme que " la sagesse n'est pas la science $^{8}$ " (Weisheit ist nicht Wissenschaft), lorsque l'on sait que, quelques années plus tard, ce dernier réajustera son tir en exprimant, de la façon suivante, le dessein d'un remarquable ouvrage :

Contribuer à ce que la philosophie approche la forme de la science (Wissenschaft) — du but [qui consiste] à pouvoir renoncer à son nom d'amour du savoir et à être savoir effectif - c'est là ce que je me suis proposé. La nécessité intérieure que le savoir soit science tient à sa nature, et c'est seulement la présentation de la philosophie elle-même qui est l'explication satisfaisante à ce propos' 9 .

Ce revirement relève d'avantage d'un approfondissement de la pensée hégélienne que d'un simple changement de cap. Il nous montre un Hegel confiant d'avoir su cerner la "nature du savoir » - seule justification de tout discours qui se prétend véritablement scientifique. Comment peut-on alors arriver à percer la nature de la scientificité? Nous entretenant du point de vue absolu à partir duquel s'élaborera sa Logique et toute sa philosophie, Hegel s'exprime ainsi à propos du "dialectique » qui doit, selon lui, s'unir $\mathrm{au}$ « spéculatif » pour atteindre adéquatement la totalité de ce qui est :

Dans ce dialectique tel qu'il est pris ici, et donc dans l'acte-de-saisir l'op-posé dans son unité, ou le positif dans le négatif, consiste le spéculatif (das Speculative). C'est le côté le plus important, mais le plus difficile pour la faculté-de-penser (Denkkraft) encore inexercée et non libre ${ }^{10}$.

Or, c'est manifestement cette capacité de saisir l'unité à même l'opposition, ou "le positif dans le négatif » (des Positiven im Negativen), qui fait défaut aux méditations qui occupent le tout jeune Hegel. À Tübingen, ce dernier ne semble pas encore arrivé à la conviction que la raison moderne puisse appréhender la totalité d'une manière satisfaisante. Posant un regard empreint d'une " nostalgie douloureuse de l'original" » sur le sentiment d'unité qu'il perçoit de ses lectures issues de la Grèce antique, Hegel se désespère du monde moderne dans les dernières lignes du Fragment : ce génie grec, écrira-t-il, « il s'est enfui de la terre ${ }^{12}$. » Il voit dans la période hellénique une vie liée où s'exprimait le monde lui-même par la bouche de ses poètes, sans entraves ni distances, dans un langage peut-être naïf, mais soustrait aux errances de l'entendement diviseur caractérisant l'avènement d'une modernité où s'exprime la douleur d'une vie désormais "brisée " et distante. L'incapacité d'appréhender l'unité dans le présent s'estompera totalement chez Hegel lors de

8. Idem.

9. Ph.G., p. $11 ;$ Ph.E., p. 71.

Io. G.W.F. Hegel, Wissenschaft der Logik: Bd. 1, Die objective Logik < désormais cité : W.L. I.>, Gesammelte Werke \# 11, Hamburg, Felix Meiner, 1978, p. 27. ; G.W.F. HEGEL, Science de la logique I : l'Être <désormais cité : S.L. I.>, trad. Bourgeois, Paris, Aubier Montaigne, 1972, p. 28-29.

I I. Tüb., p. 29 ; Frag., p. 29.

I2. Tüb. ; Frag., trad. mod., p. 297. Hegel nous dit : «[...] er ist von der Erde entflohen.» Le «er ist» dont il est question exprime le «génie» (der Genius) grec et non l' «esprit» (der Geist) comme le laisse entendre la traduction de Legros. 


\section{4 · Philosophiques / Printemps 1999}

son séjour à Jena, par la découverte de ce qu'il nommera le «principe supérieur des temps modernes que les Anciens, que Platon ne connaissaient pas ${ }^{13}$. "C'est bien à l'idée d'un progrès de la pensée moderne sur la pensée antique que nous ouvrent ces propos de Hegel, progrès qui ne se dévoile qu'à la condition que l'humanité ne se contente pas uniquement du mode de connaissance spécifique aux «Anciens ». Dès sa jeunesse, Hegel avait pourtant pressenti que le plein resplendissement de la totalité, ou l'habitation de cette "maisonnette » dont nous parle le fragment nostalgique, ne pouvait passer par l'emploi de "formules toutes faites ", que ce havre exigeait plutôt le labeur que demande un investissement de "soi ». Tirée d'un texte qui n'arrivera malheureusement pas à relier les métaphores hégéliennes à la réalité contemporaine, cette mise en garde contre les « agrégats de connaissances " nous le révèle :

Celui qui se contente de copier cet édifice universel, qui n'en recueille pas ce qui lui sert, qui ne construit pas en lui-même et par lui-même sa propre maisonnette, un chez-soi (einheimisch) avec son colombage et sa charpente, dont les pierres ont été sinon travaillées par lui-même, du moins assorties ou tournées dans ses mains, celui-là est un homme qui s'attache à la lettre, qui n'a pas vécu sa propre vie et tissé lui-même son propre caractère. Celui qui se construit un palais sur le modèle de cette grande maison, y vit comme Louis XIV à Versailles, connaît à peine tous les appartements qu'il possède et n'en occupe qu'un tout petit cabinet - un père de famille en revanche, dans sa maisonnette ancestrale est partout mieux au courant, pour rendre compte de l'emploi et de l'histoire de chaque vis et de chaque coffret ${ }^{14}$.

La Phénoménologie de l'Esprit reprendra elle aussi, à sa manière, ce constat de jeunesse en soutenant que «le bien-connu en général, pour la raison qu'il est bien-connu, n'est pas connu ${ }^{15} »$. Mais elle pousse aussi plus loin l'avertissement juvénile en l'insérant dans le sillage d'une thématique spirituelle maintenant articulée par la nature même de la science :

Parce que la substance de l'individu, parce que l'esprit-du-monde a eu la patience de parcourir ces formes dans la longueur et l'extension du temps et d'entreprendre le travail prodigieux de l'histoire du monde, et parce qu'il ne pouvait atteindre par un moindre [travail] la conscience sur soi, l'individu ne peut certes concevoir sa substance par moins de [travail $]^{16}$.

Mais, qu'entendre par l'expression « esprit du monde » (der Weltgeist), et qu'en est-il de ce travail auquel l'individu doit se soumettre? C'est ce qu'il nous incombe d'éclairer, en nous interrogeant d'abord sur la nature de l'entreprise hégélienne dans le but de répondre à la question concernant cet « esprit du monde ", pour ensuite transiter vers l'itinéraire de la Phénoménologie de

I3. G.W.F. Hegel, Jenaer Systementwürfe III, Gesammelte Werke \#8, Hamburg, Felix Meiner, 1975, p. 251 ; G.W.F. Hegel, La philosophie de l'esprit (1805), trad. Planty-Bonjour, Paris, PUF, p. 95.

I4. Tüb., p. 17 ; Frag., p. 280.

I 5. Ph.G., p. $26 ;$ Ph.E., p. 92.

I6. Ph.G., p. 25 ; Ph.E., p. 91. 
l'Esprit. Cette dernière étape nous permettra de définir la nature et l'orientation de ce labeur par lequel l'homme, nous dit Hegel, doit nécessairement passer s'il aspire au « concevoir substantiel » qui le mènera chez lui.

\section{Le projet hégélien}

Tous les écrits de Hegel tendent à confirmer la thèse selon laquelle la totalité, nommée à la fois Substance, Esprit, Absolu, ou Dieu, aurait eu l'impulsion de se particulariser pour que puisse naître un monde sensé. Depuis 1807 , avec la publication de la Phénoménologie de l'Esprit, ce Tout nous est accessible dans un Savoir qui le dévoile absolument. L'idée qui a poussé Hegel à adopter cette façon de voir réside dans sa constatation de l'insuffisance du maintien d'une totalité close, c'est-à-dire sans différence, pour qu'apparaisse le sens. Il est en effet illusoire de penser que je pourrais atteindre ce que l'on appelle généralement la "conscience de soi" ", si l'" arbre » et la " maison », pour reprendre des exemples employés par Hegel dans la Phénoménologie, n'étaient pas là pour s'opposer à moi-même en me donnant ainsi la chance de me définir comme singularité. Lisons Hegel qui nous explique comment s'articulent les différents moments qui ont animé, et continuent d'animer, le sens qui s'exhibe dans le monde que nous connaissons :

L'œil de l'esprit dut souffrir contrainte pour se trouver ramené au terrestre et maintenu près de lui ; et il fallut un long temps pour introduire à grand labeur, dans [le] trouble et [la] confusion où se trouvait le sens de l'en-deçà, cette clarté qui n'était que le fait du supra-terrestre, et pour rendre intéressante et valable l'attention à ce qui est présent comme tel, [attention] que l'on nomma expérience ${ }^{18}$.

L'on reconnaît sans trop de peine ici la référence imagée du philosophe allemand à la philosophie platonicienne, sous la métaphore de l'œil de l'esprit situé « au ciel ». Cette image, destinée à s'acheminer vers la philosophie de l'Aufklärung, atteint d'une certaine façon sa limitation sous la plume de Kant, pour qui l'esprit doit être complètement « ramené au terrestre ». À l'instar du moment grec déterminé par une unité immédiate de l'homme et du monde intuitionnée dans la sphère de l'objectivité — c'est bien chez Platon l'objet qui, en quelque sorte, nous reflète l'idée dite céleste - le kantisme prend une " tournure opposée » et radicalement subjective. Il y a bien dans ce second

I7. Ici, le terme «conscience de soi» est employé dans le sens bien précis auquel réfère la locution allemande «Bewußteyn von sich» favorisée par Hegel dans le chapitre 5 de la Phénoménologie (Cf. Ph.G., p. 234 ; Ph.E., p.397), c'est-à-dire celui du moment particulier où la conscience est inconsciente de l'abstraction qui l'anime. Le terme "Selbstbewußtseyn", traduit dans ce texte, à la manière de Labarrière, par «autoconscience», vient pour sa part corriger le caractère abstrait de l'expression «Bewußteyn von sich». Malgré beaucoup de protestations chez les commentateurs de Hegel, je persiste à croire que l'abandon de «conscience de soi» pour «autoconscience» est justifié lorsqu'il s'agit de du terme «Selbstbewußtseyn». Les explications de Labarrière sont convaincantes : "par rapport à "conscience de soi», "autoconscience» est donc requis chaque fois que ce vocable simple désigne l'identité processuelle entre le Je et le Soi.» (Cf. P.-J., Labarrière, Présentation, Ph.E., p. 56).

I 8. Ph.G., p. 13 ; Ph.E., p. 73-74. 
moment de détermination de la totalité une perte de l'objectivité qui caractérisait la splendeur de l'hellénisme, souvent qualifié de " miracle grec » où l'on suppose que l'homme se sentait pleinement chez lui dans le monde. Mais ce moment particulier se présente sous la formule d'un "manque à gagner »; car, contrairement à ce que pouvait penser Platon, Kant savait que l'objet qui lui faisait face était son objet. Dans sa Logique, Hegel souligne justement la spécificité du kantisme comme ce qui ouvre la voie au dernier moment qui nous mène au plein resplendissement de l'absolu :

Seulement cette tournure qu'a prise le connaître et qui apparaît comme une perte et un retour en arrière, a pour fondement l'aspect plus profond sur quoi repose en général l'élévation de la raison dans l'esprit plus élevé de la philosophie moderne ${ }^{19}$.

La "philosophie moderne ", comme notre auteur allemand aime l'appeler, est celle en qui se recueille l'esprit total qui a longuement mûri au cours des siècles. Hegel saisit la possibilité d'aller encore plus loin que Platon et Kant en décelant la tendance spirituelle de son époque comme la figuration d'un cercle exhibant l'Absolu qui passe par « l'impératif du contraire » d'un kantisme trop enraciné dans le terrestre, qui exige une tournure de l'Esprit dont le regard sera "ramené vers le ciel ». La suite d'un passage de la Phénoménologie précédemment cité l'illustre en ces termes :

Maintenant, ce qui paraît présent-là est l'impératif du contraire (die Noth des Gegentheils), le sens [paraît] à ce point fortement enraciné dans le terrestre qu'il est besoin d'une égale violence pour l'élever par delà ${ }^{20}$.

C'est finalement avec l'aide de ce que nous révèle la religion, que nous pouvons justement définir comme le « regard de l'esprit tourné vers le ciel », que le Tout vient à s'enrichir dans la modernité pour se montrer intégralement :

Le beau, [le] saint, [l']éternel, la religion et [l']amour sont l'appât (der Köder) qui se trouve exigé pour éveiller l'envie de mordre, ce n'est pas le concept mais l'extase, non de la Chose la nécessité qui froidement procède mais l'enthousiasme bouillonnant qui doit être l'attitude et [l']expansion portant plus avant la richesse de la substance ${ }^{21}$.

Mais en quoi la religion vient-elle " pousser plus avant la détermination substantielle ", tout en ramenant l'homme chez lui? De quelle manière se produit cet enrichissement synonyme d'un retour au point de départ du sens? C'est ce qu'entend dévoiler Hegel dans son premier grand ouvrage systématique en nous présentant le Savoir absolu qui expose dans son intégralité la totalité comme « Esprit ». L'Esprit qui, toujours selon lui, constitue « le concept le plus sublime, et qui appartient au temps moderne et à sa religion. ${ }^{22}$ »

I9. W.L. I., p. 17 ; S.L. I., p. 13-14.

2O. Ph.G., p. 13 ; Ph.E., p. $73-74$

2I. Ph.G., p. $13 ;$ Ph.E., p. 73.

22. Ph.G., p. 22 ; Ph.E., p. 87. 


\section{La Phénoménologie de l'Esprit}

Dans son Encyclopédie des sciences philosophiques, Hegel nous dit que la meilleure façon d'approcher l'absolu est d'en parler en terme d'esprit : "L'absolu est l'esprit (Geist) : c'est là la définition la plus haute de l'absolu²3. » D'une façon générale, Hegel définit l'esprit comme action, énergie (au sens fort du terme grec Evé́ $\rho \gamma_{\varepsilon}(\alpha)$, l'activité par laquelle s'exprime la totalité du réel. Replacé dans le contexte du projet hégélien, l'Esprit devient le symbole même de la nécessité d'une fragmentation du réel, de la perte de l'égalité initiale afin qu'un monde puisse se dire dans le sens. Hegel nous dira que le Savoir absolu, ou l'Esprit total, "c'est seulement cette égalité se rétablissant ${ }^{24}$ ». Une égalité qui ne sera plus immédiate mais qui, au contraire, est maintenant complètement médiatisée par les différentes formes qu'a revêtues l'Esprit en se concrétisant. La question longtemps demeurée sans réponse dont le Savoir absolu hégélien tente de nous affranchir peut se formuler ainsi : par quel moyen la dichotomie entre le sujet et l'objet, finalement entre le moi et le monde, peut-elle se résorber? Posée autrement, comment l' " autre » peut-il vraiment revenir au « même »?

Hegel affirme que les moments qui ont animé la réalisation de l'Esprit sont conservés dans le réel, par ce qu'il appelle l' "in-tériorisation ${ }^{25}$ " (ErInnerung). Il déclare aussi que le développement de la conscience humaine qui s'éveille peu à peu au savoir est en tout point identique au mouvement qui a poussé la totalité à se déterminer au fil des siècles. La Phénoménologie de l'Esprit est la "science de l'expérience de la conscience ${ }^{26}$ ", qui se propose la lourde tâche de présenter le chemin qui " passe à travers toutes les formes de la relation de la conscience à l'objet ${ }^{27}$ » — formes qui, en raison de ce que nous venons de dire, sont individuellement définies par Hegel comme figures de l'Esprit, ou comme apparition de la totalité sous la forme d'une de ses déterminations particulières. La conscience s'offre donc, pour Hegel, comme l'élément qui nous permet d'entretenir une relation privilégiée avec l'Absolu spirituel, en nous donnant la chance de refaire à notre compte, comme pour en "digérer ${ }^{28}$ » et en activer le sens, le chemin qui illustre l'histoire de la totalité. Cette histoire est toujours en passe, depuis 1807, de rayonner absolument par un savoir : "Ainsi chaque singulier parcourt-il aussi les degrés de culture de l'esprit universel, mais comme des figures que l'esprit a déjà quittées, comme les degrés d'un chemin qui est travaillé et aplani ${ }^{29}$. »Le Savoir absolu exige par contre un profond « retournement de la conscience

23. G.W.F. Hegel, Enzyklopädie der philosophischen Wissenschaften im Grundrisse <désormais cité : Enz.III>, Gesammelte Werke \# 19, Hamburg, Felix Meiner, $\mathbb{S} 384$, p. 290 ; G.W.F. HEGEL, Encyclopédie des sciences philosophiques III <désormais cité : Enc. III.>, trad. Bourgeois, Paris, Vrin, 1988, $\$ 304$, p. 99.

24. "[...] nur diese sich wiederherstellende Gleichheit». Ph.G., p. 18 ; Ph.E., p. 81-82.

25. Ph.G., p. 433 ; Ph.E., p. 694.

26. Ph.G., p. 62 ; Ph.E., p. 147.

27. W.L.I., p. 20 ; S.L.I., p.17.

28. "Verdauen", Ph.G., p. 433 ; Ph.E., p. 693.

29. Ph.G., p. 25 ; Ph.E., p. 90. 
elle-même ${ }^{30}$ ", qui dépasse largement son registre de compréhension, c'est-àdire, comme le souligne Hegel, qui dépend d'un mouvement qui «ne se présente pas pour la conscience qui est comprise dans l'expérience ellemême $^{31}$ ». Pour prendre conscience de la manière dont se réalise le Savoir absolu hégélien, et du même coup éclairer de quelle façon la religion nous aide à rentrer chez nous, attardons-nous quelque peu à définir la spécificité de cette conscience dont il est question.

\section{La conscience humaine comme cercle qui s'ignore}

Le Savoir absolu est enraciné à l'intérieur et à l'extérieur de la conscience humaine. Cette affirmation paradoxale tient à la façon de voir fondamentalement « duelle » et " linéaire " qui caractérise la conscience pour Hegel. " Duelle ", au sens où son savoir consiste " en un savoir de choses ob-jectives en opposition à soi-même, et de soi-même en opposition à elles ${ }^{32}$. » Il s'agit donc d'un savoir partiel et pas absolu, qui laisse toujours subsister quelque chose d'autre que soi-même dans sa propre définition. "Linéaire ", parce que, nous dit Hegel, la conscience ignore que « ce qui paraît survenir hors d'elle, être une activité contre elle, est son agir propre33 ». Ce double constat nous mène à l'unique conclusion, si l'on pose la possibilité, comme le fait Hegel, que puisse resplendir l'Absolu, que la conscience doit mourir à elle-même pour qu'apparaisse un Tout où l'" autre » est absolument identifié au « même ». Lisons-le :

En se propulsant vers son existence vraie, elle [scil. la conscience] atteindra un point où elle se dépouille de son apparence d'être affectée par [quelque chose d']étranger qui n'est que pour elle et comme quelque chose d'autre, ou bien où le phénomène devient égal à l'essence, [où] sa présentation du coup coïncide avec justement ce point de la science proprement dite de l'esprit, et finalement, en saisissant elle-même cette sienne essence, elle désignera la nature du savoir absolu lui-même ${ }^{34}$.

Si l'on s'accorde sur le fait incontestable que l'hégélianisme nous enseigne que la conscience se définit par la constante présence de quelque chose qui demeure " étranger pour elle ", l'on comprendra que, lorsqu'elle se "dépouille de cette apparence ", la conscience périt en perdant pour ainsi dire ce qui la détermine comme telle. Cette mise à mort participera en même temps à la transformation de ce que l'on peut appeler la "linéarité

30. "[...] eineUmkehrung des Bewußtseyns selbst.» Nous rendons le mot allemand «Umkehrung» par le terme français «retournement», et non par «conversion» comme le font Labarrière/Jarczyk. Cette traduction éclaire, d'après nous, beaucoup plus l'enjeu fondamentalement circulaire de la totalité qui s'exhibe dans la Phénoménologie lorsque la conscience devient Savoir absolu. Cf. Ph.G., p. 61 ; Ph.E., (trad.modifiée) p. 144.

3 I. Ph.G., p. 61 ; Ph.E., p. 145.

32. Ph.G., p. 23 ; Ph.E., p. 88.

33. Ph.G., p. 29 ; Ph.E., p. 97.

34. Ph.G., p. 61-62; Ph.E., p. 145. 
conscientielle », en " circularité spirituelle absolue ». Hegel nous parlera dans sa Logique, pour illustrer ce mouvement, du « cercle de la ligne qui exprime l'avancée de la science ${ }^{35}$. » Afin de saisir comment vient à s'effectuer concrètement la figuration de ce cercle, essayons de voir de quelle manière la conscience est poussée à se tourner sur elle-même lorsqu'elle va au bout de son expérience savante, en suivant le chemin tracé par l'Esprit absolu.

La dernière section de la Phénoménologie de l'Esprit, le Savoir absolu, laisse entendre que la "dualité » que l'on retrouve dans la réalité s'exprime à son apogée pour la conscience sous l'opposition que représente la " philosophie non accomplie ", qui trouve son aboutissement dans la section Esprit, et la religion " non comprise ", abordée dans les chapitres rassemblés sous le titre Religion $^{36}$. Dans cette approche dualiste, l'attitude philosophique devient synonyme d'une "passivité » misant sur la raison qui conduit la conscience à se savoir « sujet absolu »; tandis que la religion, pour sa part, nous propose une attitude foncièrement "active ", somme toute irraisonnée, d'où échoit un savoir " objectif » de la conscience. Ces deux attitudes, passive et active, devront s'unir pour que l'Absolu puisse se dire dans un savoir où rien n'est en reste. Refaisons brièvement le mouvement de la conscience qui tourne en Savoir absolu, en ne suivant que les grands moments qui en jonchent la progression.

C'est sous le signe de la "passivité » que s'engage tout d'abord la conscience dans l'expérience du monde, se laissant imbiber par ce sens qui habite toujours déjà la réalité lorsque l'homme y apparaît, assimilant les fruits de l'histoire de la philosophie, emboîtant finalement de la sorte ses propres pas dans les traces de l'Esprit absolu. Hegel illustre de cette manière le début de ce fantastique voyage :

Le savoir qui d'abord ou immédiatement est notre objet ne peut être nul autre que celui qui lui-même est savoir immédiat, savoir de l'immédiat ou de [l']étant. Nous avons à nous comporter de façon tout aussi immédiate, ou réceptive (aufnehmend), donc à ne rien changer en lui tel qu'il se propose, et à maintenir le concevoir à l'écart de l'appréhender ${ }^{37}$.

Ici, nous découvrons et appréhendons un chez-soi. Mais nous n'en sommes qu'aux préparatifs du périple qui devra nécessairement nous pousser au dehors de notre demeure afin de pouvoir répondre de chaque élément constitutif du lieu qui nous a vu naître. L'âge grec, comme nous l'avons déjà mentionné, renvoie l'image de ce moment remarquable où l'homme est chez soi. Il tient pour nous, Modernes, du domaine de notre enfance spirituelle. C'est ce vers quoi peuvent tendre ces paroles encore actuelles de Hegel :

[...] nous verrons ce qui dans les époques antérieures occupait l'esprit mûr des hommes abaissé à des connaissances, des exercices et même des jeux de

35. W.L. I., p. 35 ; S.L. I., p. 43.

36. C'est ce que nous laissent entendre ces paroles tirées du Savoir absolu de la Phénoménologie: "Cette réconciliation de la conscience avec l'autoconscience (Selbstbewußtseyn) se montre par là amenée à effet à partir d'un double aspect». Ph.G., p. 425 ; Ph.E., p. 678.

37. Ph.G., p. 63 ; Ph.E., p. 147. 
l'enfance, et, dans le progrès pédagogique, reconnaîtrons, reproduite dans une silhouette, l'histoire de la culture (Bildung) du monde ${ }^{38}$.

Le mode de l' « appréhender grec », caractérisant l'instance où les choses se dévoilaient " en soi » librement à nous, fit place, il faut en convenir en analysant l'histoire de la pensée, au «concevoir abstrait » du kantisme. Dans cet ordre d'idées, Kant demeure pour la " conscience linéaire " plus près de nous que Platon ou Aristote. L'adage suivant de la Critique de la raison pure est de ce fait, " pour elle », plus familier que les théories et sentiments relatifs à la Grèce antique : "nous ne connaissons a priori des choses que ce que nous y mettons nous-mêmes ${ }^{39}$. " Par cette détermination du savoir, on le voit, l'objectivité disparaît, s'efface devant la souveraineté de la subjectivité humaine. La « brisure » de la " belle totalité grecque » est ainsi consommée ; la vérité n'y est que " pour soi » et devient plurielle en s'exprimant par des concepts : " [l]a chose est Je [...] ; elle n'est rien en soi ${ }^{\circ}{ }^{\circ}$ ». Mais le "Concept » de ces « concepts » demeure par contre dans l'ombre. On ne peut qu'acquiescer au fait que le kantisme ait échoué à exprimer absolument la totalité. Hegel nous explique que si le savoir kantien demeure abstrait, c'est parce qu'il dénote un retrait de la totalité vivante, un repliement sur soi de l'homme qui s'y conforme, que la conscience qui l'exprime "demeure dans l'universalité de la pensée, se comporte comme [conscience] qui-saisit (als auffassendes), et son opération première est seulement le jugement ${ }^{41}$ ", que son action ne consiste qu'à faire " jouer l'opération dans l'intérieur ${ }^{42}$ ». Or, ce savoir partiel, que Hegel nomme aussi " belle âme » (die schöne Seele) doit, s'il aspire à la vérité, renoncer à son abstraction et se confier à l'élément objectif de la réalité. C'est à ce moment bien particulier de la quête du savoir qu'intervient l'aide de la religion, exprimée dans des paroles hégéliennes précédemment citées.

L' « objectivité » pour la conscience est présentée par l'élément religieux, qui, sous le puissant symbolisme du Dieu «personnel » de la religion chrétienne, que Hegel qualifie de " religion moderne », relègue l'homme au simple rang de "chose » ou de «créature » devant le «sujet divin" "créateur". Mais même cette région de la conscience que l'on nomme religion, qui exhale l'objectivité, ne pourra exprimer absolument le monde, puisque la subjectivité y est extérieure : "Dieu lui-même est mort ${ }^{43}$." Tout se passe finalement comme si la Phénoménologie de l'Esprit soutenait qu'il existe deux principales options qui s'offrent immédiatement à nous en matière de

38. Ph.G., p. 25 ; Ph.E., p. 90-91.

39. I. Kant, Kritik der reinen Vernunft, Kants Werke \# 3, Berlin, Walter de Gruyter \& Co., 1968, p. 13 ; E. Kant, Critique de la raison pure, trad. Tremesaygues/Pacaud, PUF, 1944, p. 19.

40. Ph.G., p. 423 ; Ph.E., p. 676.

4I. Ph.G., p. 357 ; Ph.E., p. 573.

42. Ph.G., p. $358 ;$ Ph.E., p. 574.

43. Cette affirmation lourde de conséquences déjà présente dans l'ouvrage de jeunesse Foi et Savoir (Glauben und Wissen) est reprise dans la Phénoménologie de l'Esprit. Ph.G., p. 419 ; Ph.E., p. 669. 
vérité et de sens, ou de " négativité motrice du réel »; que l'on peut affirmer que c'est Dieu en tant que "négativité pure dans la forme de l'universalité44 » qui anime le monde et le rend sensé, comme dans la religion, ou bien décréter que c'est l'homme savant, "cette même [négativité] dans la forme de la singularité45 ", comme dans la philosophie pré-hégélienne. Mais le Savoir absolu hégélien consiste à prendre conscience que le choix de l'une de ces deux options ne peut arriver à exprimer adéquatement l'absolu. L'une manque d'objectivité, l'autre de subjectivité. C'est à cette mort inévitable de la conscience, qui ne peut entrevoir philosophie et religion que comme des éléments séparés, que nous convie le Savoir absolu hégélien.

\section{La concrétisation du Savoir absolu}

En s'appuyant sur la détermination de l'esprit exprimée dans la religion, qui illustre en fait une conscience au terme de sa course, Hegel affirmera, dans la section consacrée au Savoir absolu, l'exigence d'un recourbement de la conscience scellant sa mise à mort. Voyons comment agit cet esprit religieux :

C'est seulement après avoir abandonné l'espoir de sursumer l'être-étranger d'une manière extérieure, i.e. étrangère, qu'il [scil. l'esprit] se tourne (wendet es sich), parce que la manière étrangère sursumée est le retour de l'autoconscience, vers lui-même, vers son monde et [son] présent propres, les découvre comme sa possession ${ }^{46}$.

Que nous dit le penseur dans ce passage, sinon que la conscience se " tourne » sur elle-même pour réintégrer ce chez-soi auquel l'humanité a dû renoncer pour goûter aux fruits de l'arbre de la connaissance en retrouvant ce qui est présent là comme "sa possession »? Ou, comme il l'anticipait dans la préface de sa première œuvre maîtresse, que la vérité est parvenue à sa « forme native (ihrer einheimischen Form), laquelle est capable d'être propriété de toute raison autoconsciente 47 » ? Hegel précise que ce retour à l'immédiateté du départ se terminera par la résurgence de la figure nommée la «belle âme ", cette intériorité qui affirmait la suprématie de la subjectivité conscientielle, qui de surcroît perdra son attitude de partialité et même son nom en devenant ce qui " abandonne son essence éternelle, est là, ou opère ${ }^{48}$. " De cette façon, l' "autre " s'identifie au " même " et exhibe un Savoir absolu, la " passive intériorité » s' « active » et s' " extériorise » dans un savoir où rien ne peut plus être mis à l'écart. Les manques relatifs à l'attitude philosophique et religieuse sont comblés sous le constat d'un Savoir total qui révèle qu'agir effectivement, c'est en fait assumer " la force de l'extériorisation, la force de se faire chose et de supporter l'être 49 ». Lorsqu'il agit de la sorte, l'homme devient le sujet qui

44. Ph.G., p. 363 ; Ph.E., p. 583.

45. Idem.

46. Ph.G., p. 430 ; Ph.E., p. 688.

47. Ph.G., p. 48 ; Ph.E., p. 126.

48. Ph.G., p. 426 ; Ph.E., p. 681.

49. Ph.G., p. 354 ; Ph.E., p. 569. 
altère lui-même sa conscience, puisque la conscience se confond initialement avec le monde qu'elle sent. Autrement dit, l'homme s'objective dans l'actualisation de la négativité qui l'habite. En transformant le donné qui détermine d'abord cette conscience, il s'identifie à l'autre qui devait demeurer étranger pour elle. L'humanité est ainsi unité du théorique et du pratique présentant une totalité vivante qui a relevé la contradiction; elle est esprit : « le savoir de soi-même dans son extériorisation ; l'essence, qui est le mouvement de garder dans son être-autre l'égalité avec soi-même ${ }^{50}$ ». Cette égalité, c'est celle qui, dans l'action " autoconsciente », proclame le sens absolu de tout ce qui est par la conviction que la réalisation de l'homme dans le monde coïncide avec la réalisation du monde dans l'homme.

Hegel a su dépasser le kantisme qui brillait encore à son époque, qui n'agissait " que dans un dire ${ }^{51}$ », avec une vérité exigeant des réponses nettes, trop nettes en fait pour englober le Tout, en convenant du fait que : " quel qu'il soit, chaque [contenu] a en lui la tache de la déterminité (Makel der Bestimmtheit) $)^{52}$ ". Cette "tache " assumée, l'humanité retrouve la "maisonnette" dont il était question dans l'écrit de Tübingen, "petite maison » qui se démarque par contre du «chez-soi » grec en ce que le lieu que l'homme habite maintenant a complètement été agencé par lui ; les pierres qui le constituent ont été "tournées dans ses propres mains". Le savoir kantien nous gardait les mains propres en nous reléguant aux affres de l'itinérance, condamnant l'homme qui s'y soustrayait à ne jamais trouver dans le monde le repos du logis. Le savoir hégélien exige plutôt des mains sales qui s'unissent au labeur de l'esprit absolu, et doivent nécessairement passer par l'attitude religieuse, pour que l'humanité arrive à habiter une demeure spirituelle où elle se sent pleinement chez elle. Heureusement pour nous, Modernes contraints à expérimenter la douleur d'une vie brisée, l'état dichotomique du savoir n'est que temporaire :

« Die Wunden des Geistes heilen, ohne daß Narben bleiben ${ }^{53} » \ldots$

50. Ph.G., p. 405 ; Ph.E., p. 646.

5I. B. Rousset, G.W.F. Hegel, Le Savoir absolu, Paris, Aubier Montaigne, 1977, p. 147.

52. Ph.G., p. 348 ; Ph.E., p. 560.

53. «Les blessures de l'esprit guérissent sans que cicatrices demeurent». Ph.G., p. 360 ; Ph.E., p. 578. 